Classification

Physics Abstracts

0.667

\title{
DEUX INSTRUMENTS POUR LA SPECTROSCOPIE RMN
}

\author{
S. F. J. $\operatorname{COX}\left({ }^{*}\right)$ et V. BOUFFARD \\ Service de Physique du Solide et de Résonance Magnétique \\ Centre d'Etudes Nucléaires de Saclay, B. P. no 2, 91190 Gif-sur-Yvette, France
}

(Reçu le 14 février 1976, révisé le 16 juin 1976, accepté le 17 juin 1976)

\begin{abstract}
Résumé. - Nous décrivons deux instruments utilisables en spectroscopie RMN dans le cas de raies larges. Le premier est une modification du $Q$-mètre classique par un dispositif à servomécanisme qui accorde automatiquement le circuit résonant à la fréquence de l'oscillateur. La stabilité du $Q$-mètre est améliorée et les dispersions électrique et magnétique sont supprimées.

Le second appareillage a trait au calcul analogique de diverses intégrales d'une raie RMN. Suivant en continu l'aire de la raie, il permet la mesure de la susceptibilité, de la polarisation dynamique et des temps de relaxation dans le référentiel du laboratoire et dans le référentiel tournant. Des variantes de cet instrument rendent possibles la mesure de l'énergie dipolaire, des temps de relaxation dipolaire et d'autres quantités telles que les moments d'ordre supérieur de la raie et la susceptibilité transverse.
\end{abstract}

\begin{abstract}
Two instruments are proposed for use in wide-line NMR spectroscopy. The first is a servomechanism, a modification of a simple $Q$-meter spectrometer which automatically tunes the resonant circuit to the oscillator frequency. The stability of the $Q$-meter is improved and both the electrical and the magnetic dispersion are suppressed.

The second device concerns the analogue calculation of various integrals of the NMR line. The basic instrument monitors continuously the area of a NMR line and has application in the measurement of susceptibility or dynamic polarization, and of nuclear Zeeman relaxation times both in the laboratory and the off-resonance rotating frames. Variants of the instrument permit the measurement of dipolar energy, dipolar relaxation times, and of other quantities such as the higher moments of the line and the transverse susceptibility.
\end{abstract}

1. Introduction. - Dans cet article sont décrits deux appareils utilisés en résonance magnétique nucléaire, en particulier pour l'observation des raies larges des solides diélectriques à basse température.

Ces instruments facilitent les mesures statiques (susceptibilité nucléaire, polarisation dynamique ou énergie dipolaire par exemple), et des mesures dynamiques telles que celles des temps de relaxation Zeeman et dipolaires ou des temps de relaxation dans le référentiel tournant.

Le premier instrument est une modification simple d'un spectromètre du type $Q$-mètre. Il utilise un servomécanisme qui accorde automatiquement le circuit de résonance électrique à la fréquence de l'oscillateur, même quand cette fréquence est balayée pour faire apparaître la raie de résonance magnétique sur l'écran d'un oscilloscope. Ceci a pour effet de supprimer la composante dispersive du signal RMN, ainsi que la ligne de base parabolique caractéristique des $Q$-mètres.

$\mathrm{Ce}$ dispositif rend les raies de résonance obtenues avec un $Q$-mètre plus propres à l'observation et aux

(*) Adresse permanente : Rutherford Laboratory Oxfordshire OX11 OQX, England. mesures quantitatives. De plus, il améliore considérablement la stabilité à long terme du spectromètre. Ce servomécanisme est décrit dans la section 2.1.

Le second appareil permet la mesure directe de la surface d'une raie d'absorption RMN (donnée par exemple par un $Q$-mètre asservi) évitant ainsi l'opération fastidieuse de simple, ou même double intégration. Il permet également l'enregistrement continu de l'évolution dans le temps de cette mesure (ainsi que celui d'autres intégrales de la raie). De par son principe même, l'instrument utilise la détection synchrone, ce qui améliore le rapport signal sur bruit des mesures, et permet l'étude des signaux faibles. Un avantage supplémentaire provient de ce que les mesures sont indépendantes de la hauteur de la ligne de base des signaux et donc insensibles aux dérives.

Le principe de l'appareil est décrit dans la section 3.1 , sa réalisation dans la section 3.2 ; un exemple de son utilisation, l'enregistrement de la relaxation dans le référentiel tournant, est donné dans la section 3.3. Des variantes proposées dans la section 3.4 permettent la mesure d'autres intégrales et, à titre d'exemple, un enregistrement de relaxation dipolaire est décrit dans la section 3.5. Les erreurs systématiques 
et la comparaison avec des méthodes digitales, sont examinées dans la section finale.

2. Accord automatique de la fréquence de résonance. - 2.1. Principe DU SERVoméCANISME. - L'ensemble de départ est un $Q$-mètre à courant constant $[1,2]$ et à circuit résonant parallèle. En fonction de la fréquence, le signal de sortie, dit ligne de base, est de forme parabolique, le maximum correspondant à la résonance électrique du circuit. Si l'on place en parallèle sur le circuit $\mathrm{LC}$, représenté dans la figure 1 , un

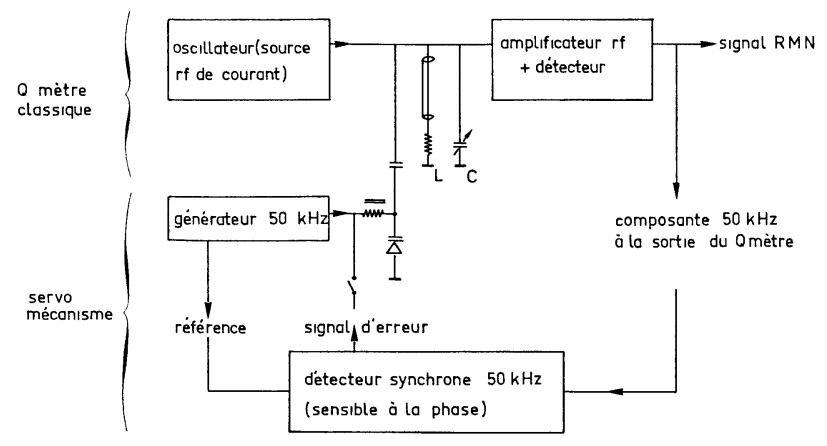

Fig. 1. - Schéma du $Q$-mètre et du servomécanisme. La bobine $\mathbf{L}$ et la combinaison parallèle de la capacité $C$ et de celle de la diode forment le circuit résonant. A cause des dispositifs cryogéniques, la bobine qui contient l'échantillon, est séparée du circuit par un câble coaxial (de longueur $n \lambda / 2$ ).

ensemble capacité et diode (du type Varicap) la parabole se déplacera en fonction de la valeur de la tension appliquée aux bornes de la diode. Cette tension sera commandée automatiquement pour que le circuit soit toujours correctement accordé, et que sa fréquence de résonance suive la fréquence du générateur de courant.

Le fonctionnement du servomécanisme est le suivant. Un signal carré à $50 \mathrm{kKz}$ (Fig. 2a), envoyé sur la diode, module la parabole entre deux enveloppes extrêmes se croisant au voisinage de leur maximum. Ce principe est illustré dans la figure $2 b$; dans la pratique, l'écart

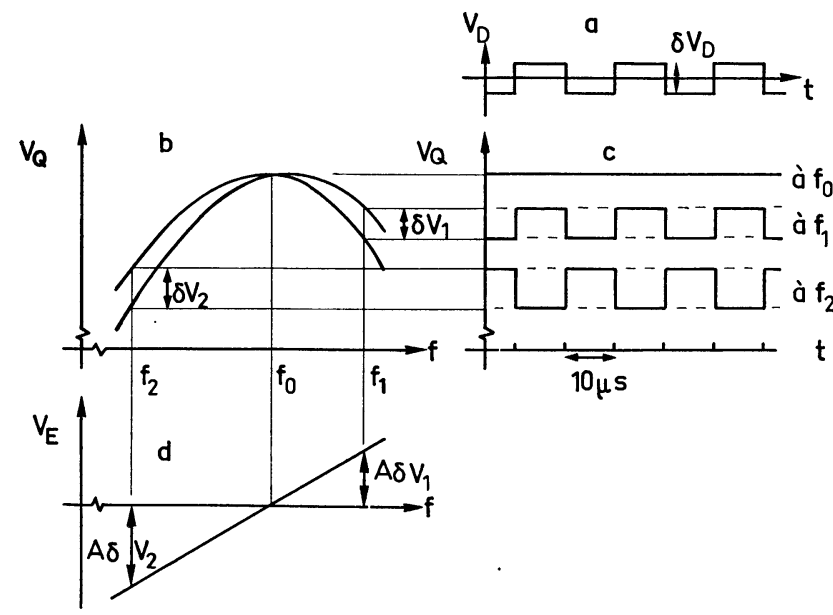

Fig. 2. - Construction du signal d'erreur du servomécanisme en boucle ouverte. de ces deux paraboles sera faible par rapport au bruit de détection et invisible sur la trace de l'écran d'un oscilloscope. De part et d'autre du point de croisement, les phases du signal à $50 \mathrm{kHz}$ sortant du $Q$-mètre sont de signe opposé (Fig. 2c). Un détecteur sensible à la phase convertit ce signal en une tension continue d'erreur (Fig. $2 d$ ) $\left({ }^{1}\right.$ ) qui est réinjectée sur la diode. Cette contre-réaction asservit la capacité équivalente du circuit de façon à donner constamment un maximum de tension à la sortie du $Q$-mètre.

La figure 3 illustre le fonctionnement du servomécanisme. Le signal enregistré est celui des protons

(b)

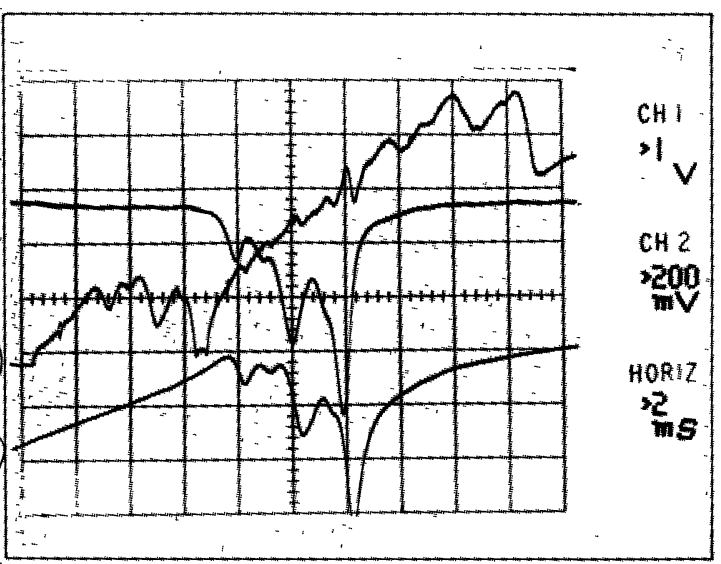

Fig. 3. - Tests du servomécanisme. a) Signal RMN sans contre réaction. $b$ ) Même signal après fermeture de la boucle de contre réaction. c) Signal d'erreur. (Raie $\mathrm{RMN}$ de ${ }^{1} \mathrm{H}$, hautement polarisée, dans le $\mathrm{La}_{2} \mathrm{Mg}_{3}\left(\mathrm{NO}_{3}\right)_{12.24} \mathrm{H}_{2} \mathrm{O}$.)

fortement polarisés par effet solide [3] à 18 kilogauss dans un échantillon de LMN dopé au Néodyme. La trace (a) montre le signal RMN à la sortie du $Q$-mètre lorsque la boucle de contre-réaction est ouverte. Le circuit résonant a été délibérément désaccordé et le signal est un mélange d'absorption et de dispersion, superposé à une ligne de base parabolique. Quand la boucle de contre-réaction est fermée sans autre réglage, la parabole s'aplatit sensiblement et le signal RMN est uniquement un signal d'absorption (trace b). (Plus exactement la contribution dispersive est réduite au deuxième ordre.) Le signal d'erreur (trace c) est dû à la fois à la dispersion électrique du circuit (droite inclinée) et à celle des spins nucléaires.

2.1.1 Calcul du gain de la boucle G. En négligeant la réponse en fréquence de l'amplificateur, le gain de boucle du servomécanisme se calcule à partir de l'équation donnant la tension de sortie du $Q$-mètre [4]

$$
V_{Q}=V_{0}\left\{1-2 Q^{2}\left(\frac{f-f_{0}}{f_{0}^{2}}\right)^{2}\right\}
$$

(1) Ce principe est semblable à celui d'une commande automatique de fréquence pour des spectromètres RPE, due à J. C. Gill (communication privée, 1965). 
ou $f$ est la fréquence du générateur et $f_{0}$ la fréquence d'accord du circuit. Celle-ci dépend de la tension de polarisation $V_{\mathbf{D}}$ appliquée aux bornes de la Varicap suivant la relation

$$
\frac{\mathrm{d} f_{0}}{\mathrm{~d} V_{\mathrm{D}}}=-\frac{f_{0}}{2 C} \frac{\mathrm{d} C}{\mathrm{~d} V_{\mathrm{D}}}
$$

Soit $\delta V_{\mathrm{D}}$ l'amplitude de modulation de $V_{\mathrm{D}}$ et $A$ le gain du détecteur synchrone. En boucle ouverte, on trouve qu'une perturbation continue $\Delta V_{\mathrm{D}}$ produit un changement $\Delta V_{\mathrm{E}}$ du signal d'erreur

$$
\Delta V_{\mathrm{E}}=A \frac{\mathrm{d}^{2} V_{Q}}{\mathrm{~d} f^{2}}\left(\frac{\mathrm{d} f_{0}}{\mathrm{~d} V_{\mathrm{D}}}\right)^{2} \delta V_{\mathrm{D}} \Delta V_{\mathrm{D}} .
$$

Le gain $G$ de la boucle est donc :

$$
G=\frac{\Delta V_{\mathrm{E}}}{\Delta V_{\mathrm{D}}}=\frac{Q^{2} A V_{0}}{C^{2}}\left(\frac{\mathrm{d} C}{\mathrm{~d} V_{\mathrm{D}}}\right)^{2} \delta V_{\mathrm{D}} .
$$

Pour notre prototype, les données relatives aux figures 2 et 3 sont les suivantes :

$$
\begin{gathered}
\frac{\mathrm{d}^{2} V}{\mathrm{~d} f^{2}}=4,4 \times 10^{-3}(\mathrm{mV})(\mathrm{kHz})^{-2} ; \\
\frac{\mathrm{d} f_{0}}{\mathrm{~d} V_{\mathrm{D}}}=6 \times 10^{-2}(\mathrm{kHz})(\mathrm{mV})^{-1} \\
\delta V_{\mathrm{D}}=10 \mathrm{mV} ; \quad A=5 \times 10^{4} \text { et } G=8 .
\end{gathered}
$$

En boucle fermée, la petite amplitude résiduelle de la parabole représente la variation avec la fréquence du gain de l'amplificateur rf accordé.

2.2 SCHÉmA vectoriel. - Sur la figure 4, on représente l'impédance du circuit accordé par le rayon

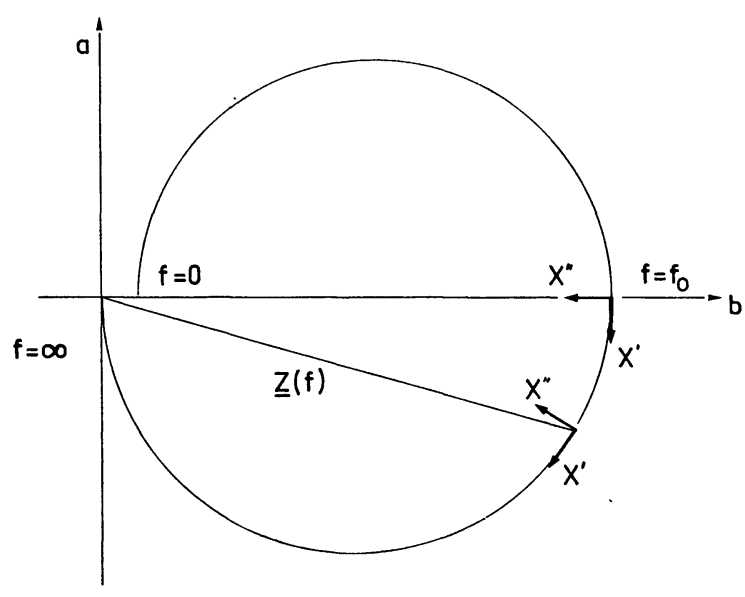

Fig. 4. - Diagramme vectoriel de l'impédance $\mathbf{Z}=\mathbf{a}+\mathrm{jb}$ d'un circuit résonant parallèle. Les composantes de $\mathbf{Z}$ dues à l'absorption $X^{\prime \prime}=4 \pi \eta Q \chi^{\prime \prime} Z$ et à la dispersion $X^{\prime}=4 \pi \eta Q \chi^{\prime} Z$ sont indiquées pour $f=f_{0}$ et $f \neq f_{0}$.

vecteur $\mathbf{Z}=a+j b$. A valeur constante des paramètres électriques $L, C, r$ (c'est-à-dire à boucle de contre réaction ouverte), l'extrémité du rayon vecteur décrit la courbe de la figure 4 quand la fréquence varie de zéro à l'infini. Dans un $Q$-mètre, le circuit est alimenté par une source rf de courant: la tension de sortie une fois détectée est donc proportionnelle à $|Z|$ c'est-àdire à la longueur du rayon vecteur. Elle est maximum pour une fréquence $f_{0}$ voisine de celle qui donne une impédance réelle [4].

Pour des signaux RMN suffisamment faibles (rapport signal sur porteuse inférieur à $10^{-1}$ ) les déplacements dus à l'absorption et à la dispersion sont orthogonaux [1]; leur disposition est représentée sur la figure 4. A la résonance électrique, la composante dispersive est perpendiculaire au rayon vecteur et contribue au signal RMN seulement au second ordre. Toutefois si le circuit résonant est désaccordé, la composante dispersive n'est plus perpendiculaire au rayon vecteur, et elle contribue au premier ordre au signal RMN.

Le servomécanisme ajuste la réactance du circuit accordé pour que $|Z|$ soit toujours maximum (par rapport à $C$ ), lorsque la boucle de contre réaction est fermée : le circuit est donc toujours à la résonance électrique. En balayage de fréquence, il reste une légère pente dans la ligne de base puisque les maxima de $|Z|$ par rapport à $C$ et à $f$ sont différents de $\Delta f \sim f_{0} / Q^{2}$. Cette pente représente l'augmentation de $Q$ avec $f$ et l'effet transformateur du câble coaxial qui, dans notre cas sépare $L$ et $C$. L'effet est minimum pour un câble le plus court possible, c'est-à-dire de longueur $\lambda / 2$ et d'impédance $Z_{0}$ proche de $2 \pi f_{0} L$.

Le temps de réponse du servomécanisme (soit l'inverse de la bande passante finale du détecteur synchrone $50 \mathrm{kHz}$ ) doit être court par rapport à la période de balayage de fréquence. (Dans notre prototype, la période de modulation est de $30 \mathrm{~ms}$, le temps de réponse de l'ordre de $300 \mu$ s.) Les changements de réactance dus à la dispersion électrique et nucléaire sont par 'conséquent corrigés en même temps. On remarque que dans le cas d'un balayage de champ magnétique, à fréquence fixe, le temps de réponse du servomécanisme peut être soit court, comme ci-dessus, soit long. Dans ce dernier cas, seules les dérives lentes d'accord sont corrigées.

2.3 Comparaison AVEC D'AUTRES SYSTÈmes. - Il existe un schéma différent de contre réaction proposé par Petricek [5] dans le cas d'un circuit résonant série. Ce schéma utilise un détecteur synchrone à la fréquence r. f. du $Q$-mètre, et est reproduit dans la figure 5 .

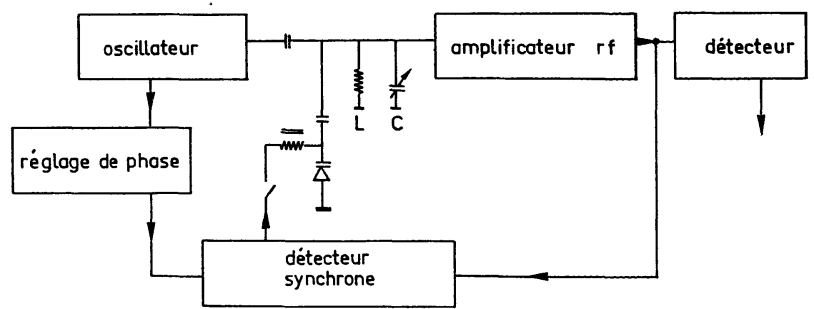

Fig. 5. - Schéma d'un autre système de contre réaction. Le détecteur synchrone peut être du type pont-diode (double balanced mixer). 
Cette contre-réaction a l'inconvénient de corriger la dispersion dans tout le système. La contribution des amplificateurs r. f. et des câbles coaxiaux auxiliaires n'est pas négligeable devant celle du circuit résonant seul, puisque l'on n'utilise pas des amplificateurs à large bande, ni des câbles très courts. Ce circuit ne serait donc pas correctement accordé pour de grandes explorations de fréquence.

Cette difficulté est évitée dans un système superhétérodyne (également pour circuit résonant série) dû à Madden [6]. Un comparateur mesure, à la fréquence intermédiaire, l'écart de phase entre le circuit qui contient l'échantillon et un cirçuit identique sans échantillon, servant de référence.

3. Mesure analogique des intégrales d'une raie d'absorption. - 3.1 MESURE DE L'AIRE D'UNE RAIE. La mesure de l'aire d'une raie RMN trouve son intérêt dans l'étude, par exemple, des susceptibilités nucléaires et de la polarisation dynamique. L'enregistrement continu de l'aire permet de plus l'observation de l'évolution de la susceptibilité, la croissance et la décroissance de la polarisation dynamique, et la mesure du temps de relaxation spin-réseau.

Un signal en dent de scie module le champ magnétique ou la fréquence $r$. f. avec une amplitude supérieure à la largeur de la raie RMN. A la sortie du $Q$-mètre, la raie d'absorption apparaît alors répétitivement (trace a de la figure 6).

A chaque cycle, un amplificateur à porte prend trois échantillons consécutifs de ce signal (trace b) : un échantillon (A) inversé de la ligne de base hors du signal $\mathrm{RMN}$ et de durée $t$ ( $\simeq 5 \mathrm{~ms}$ par exemple), un

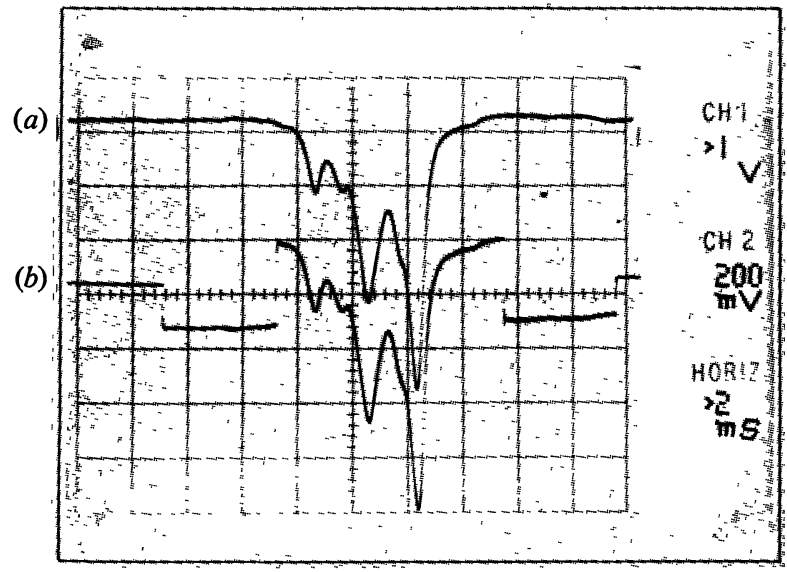

Fig. 6. - Mesure de l'aire d'une raie RMN. La trace a de l'oscillogramme est le signal de sortie d'un $Q$-mètre asservi (voir la figure $3 b$ ). La trace $b$ représente le signal intermédiaire qui comprend les trois échantillons : l'échantillon central avec la raie de résonance, et les deux échantillons inversés de la ligne de base de chaque côté. (Par comparaison de l'aire de cette raie, c'est-à-dire le signal de sortie du moyenneur, avec celle du signal d'équilibre thermique, la polarisation est estimée à $92 \% \pm 5 \%$.) Raie $\mathrm{RMN}$ de ${ }^{1} \mathrm{H}$ dans le

$$
\left(\mathrm{La}_{1-x}, \mathrm{Nd}_{x}\right)_{2} \mathrm{Mg}_{3}\left(\mathrm{NO}_{3}\right)_{12} .24 \mathrm{H}_{2} \mathrm{O} \text {, }
$$

avec $x \sim 1 \% ; \omega_{0}=80 \mathrm{MHz}, \mathrm{d} \omega=20 \mathrm{kHz}$ par carreau. échantillon (B) non inversé de durée $2 t$ qui contient le signal entier, et un deuxième échantillon $(C)$ de la ligne de base inversé, de durée $t$. Le signal résultant dit signal intermédiaire, est moyenné sur un grand nombre de cycles avec une constante de temps appropriée (de $100 \mathrm{~ms}$ à $10 \mathrm{~s}$ par exemple), pour en extraire sa composante continue. La tension de sortie du moyenneur est donc proportionnelle à la surface de la raie RMN. Elle est de plus insensible aux dérives de la ligne de base puisque cette dernière est soustraite du signal d'absorption à chaque cycle (avant de prendre la moyenne).

Cet échantillonnage est donc particulièrement intéressant pour les $Q$-mètres à circuit résonant parallèle dans le cas de signaux faibles (rapport signal sur porteuse inférieur à $10^{-2}$ par exemple). De plus, en prenant la moyenne sur un grand nombre de cycles, le rapport signal sur bruit est amélioré par un facteur de l'ordre de

$$
\delta\left(B_{Q} / B_{M}\right)^{1 / 2}
$$

avec $\delta=\tau / 4 t$, où $\tau$ désigne le temps de passage sur la raie et $4 t$ le temps d'échantillonnage par cycle. $B_{Q}$ est la bande passante du $Q$-mètre, $B_{M}$ la bande passante $\mathrm{du}$ moyenneur.

3.2 DeSCRIPTION DES CIRCUITS. - La figure $7 a$ montre un schéma simplifié de l'appareil. Les deux étages utilisent chacun un amplificateur opérationnel (circuit intégré) avec un montage standard. Dans l'étage échantillonneur, les interrupteurs $S_{1}$ et $S_{2}$ s'ouvrent et se ferment suivant les signaux illustrés au bas de la figure $7 b$. L'étage se comporte comme un amplificateur non inverseur $\left(S_{1}\right.$ fermé, $S_{2}$ ouvert, gain $=+r_{2} / r_{1}$ pendant l'intervalle $B$, et comme un amplificateur inverseur $\left(S_{1}\right.$ ouvert, $S_{2}$ fermé, gain $=-r_{2} / r_{1}$ à condition que $\left.r_{3}=2 r_{1} r_{2} /\left(2 r_{1}+r_{2}\right)\right)$ pendant les intervalles $A$ et $C$. Le signal intermédiaire dont un exemple est donné sur la figure $6 b$ est ensuite moyenné dans un étage d'amplification continue de gain $-r_{5} / r_{4}$ et de constante de temps $r_{5} C$.

3.3 Mesure Du temps DE RElaxation DaNS LE RÉFÉRENTIEL TOURNANT. - Cet instrument peut être utilisé par exemple pour la mesure de la polarisation dynamique (par comparaison de l'aire du signal polarisé avec celle du signal d'équilibre thermique) $\left({ }^{2}\right)$, ou pour la mesure du temps de polarisation et de relaxation (Zeeman nucléaire-réseau).

On remarquera simplement ici, que pour des polarisations nucléaires élevées, la forme de la raie $\mathrm{RMN}$ change en fonction de la polarisation. Il est donc important pour la mesure de surface que le balayage de

(2) L'instrument est actuellement utilisé pour la mesure de polarisation dynamique dans ce laboratoire, au Rutherford Laboratory Oxfordshire, Angleterre et à l'Institut Laue-Langevin, Grenoble, France [7]. A ce propos, un système digital qui totalise le résultat de $N=100$ à 1000 cycles, avec soustraction de la ligne de base a été proposé indépendamment par Banks [8]. 
(a)

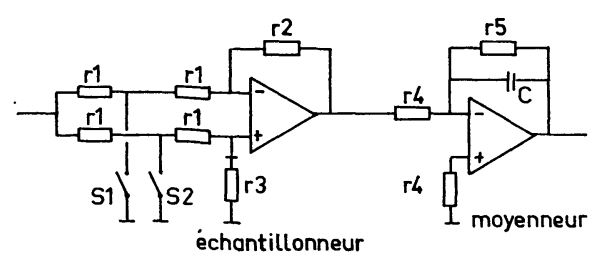

(b)

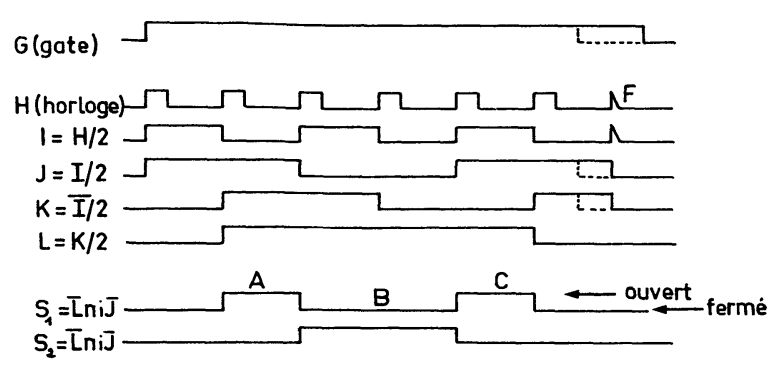

(c)

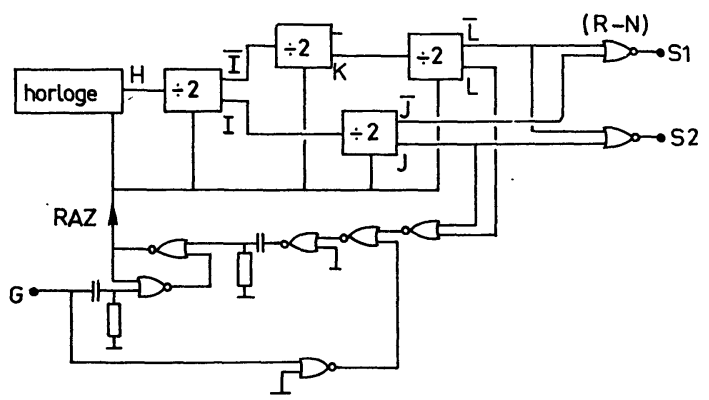

FIG. 7. - Réalisation de l'intégrateur synchrone. a) Partie analogique. $b$ ) Génération des signaux de commande des interrupteurs. c) Partie digitale générateur $(\mathrm{R}-\mathbf{N}=$ réunion négation). Le cycle est déclanché par la montée du signal gate (qui représente par éxemple, la durée de la rampe linéaire de l'oscilloscope) ; les circuits sont remis en attente soit par le front descendant du signal gate, soit automatiquement à la fin de la séquence (point $\mathrm{F}$ ).

fréquence soit bien linéaire, et suffisamment large pour inclure les ailes de la raie dans l'échantillon central (voir § 3.6).

Comme exemple d'application plus élaborée, nous allons décrire la mesure du temps de relaxation dans le référentiel tournant [1] (ou de saturation loin de la résonance, selon le système étudié). L'expérience consiste à enregistrer la décroissance de la polarisation nucléaire en présence d'une forte irradiation $r$. $f$. en dehors de la raie $R M N$. L'observation de la raie avec un $Q$-mètre (à faible puissance r. f. qui ne sature pas la résonance) et l'enregistrement de sa surface, demeurent inchangés. On ajoute en outre une séquence d'impulsions de puissance r. f. avec un oscillateur indépendant du $Q$-mètre. Le niveau de sortie de l'oscillateur est piloté en synchronisme avec le cycle de mesure de telle façon que les impulsions tombent dans le temps mort entre chaque cycle de l'échantillonneur (lorsque $S_{1}$ et $S_{2}$ ont tous les deux fermés, voir Fig. 7). L'observation et l'enregistrement de la décroissance de l'aimantation sont donc possibles pendant l'irradiation. Il est cependant nécessaire de s'assurer que le spectre de Fourier de l'onde d'irradiation modulée, ne possède pas de composante notable au voisinage de la fréquence de résonance nucléaire. Un enregistrement de démonstration est donné dans la figure 8 . Il représente un signal de ${ }^{19} \mathrm{~F}$ dans un échan-

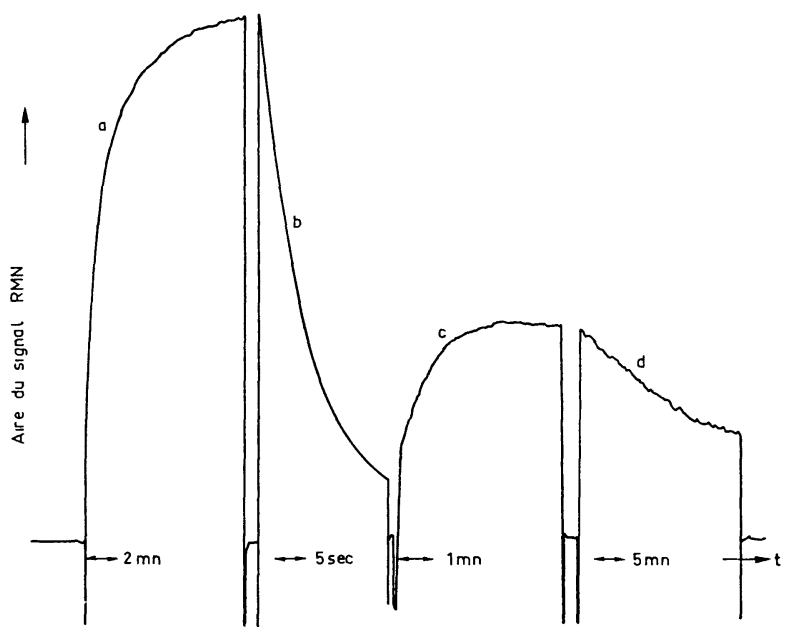

FIG. 8. - Enregistrement continu de l'aire d'une raie RMN de fluor ${ }^{19} \mathrm{~F}$ dans des expériences diverses avec du fluorure de lithium (LiF dopé avec des centres $\mathrm{F}$ à $25 \mathrm{kG}$ et $0,6 \mathrm{~K}$ ). a) Polarisation dynamique ; $b$ ) Relaxation dans le référentiel tournant; c) "Regonflage spontané » du signal par mélange thermique avec une deuxième espèce nucléaire $[9]$; $d$ ) Relaxation spinréseau.

tillon de LiF dopé avec des centres $F$, et polarisé par effet solide à $25 \mathrm{kG}$ et $0,6 \mathrm{~K}$. Les quatres phases $a, b, c$ et $d$ sont successivement : la polarisation dynamique, la relaxation dans le référentiel tournant, le regonflage spontané et la relaxation spin-réseau. Entre les différentes phases, le champ magnétique a été décalé. Le signal disparaît du système d'échantillonnage et l'on obtient sur l'enregistrement la ligne de base.

3.4 Mesures D'AUTRES intéGrales. - Dans l'instrument de base, ainsi décrit, la fréquence de l'oscillateur est balayée linéairement dans le temps, et l'intégrale par rapport au temps du signal de sortie du spectromètre est proportionnelle à la surface de la raie $\mathbf{R M N}$

$$
\int \chi^{\prime \prime}(\omega) \mathrm{d} \omega \propto \int V(t) \mathrm{d} t
$$

Ce principe peut être généralisé en utilisant d'autres modes de balayage de la fréquence, afin d'obtenir différentes intégrales de la raie, intéressantes pour le physicien : notamment l'énergie dipolaire des spins qui est proportionnelle au premier moment de la raie RMN par rapport à la fréquence de Larmor [10] :

$$
E_{\mathbf{D}} \propto \int \chi^{\prime \prime}(\omega)\left(\omega-\omega_{0}\right) \mathrm{d} \omega,
$$


ou la susceptibilité transversale qui est égale à la dispersion au centre de la raie :

$$
\chi_{\perp}=\chi^{\prime \prime}\left(\omega_{0}\right)=\mathfrak{\int} \int \chi^{\prime \prime}(\omega)\left(\omega-\omega_{0}\right)^{-1} \mathrm{~d} \omega
$$

La première intégrale $\left(E_{\mathrm{D}}\right)$ est toujours possible à effectuer en calcul analogique quelle que soit la forme de la raie $\chi^{\prime \prime}(\omega)$. La seconde $\left(\chi_{\perp}\right)$ n'est possible en pratique que dans le cas d'une raie de forme antisymétrique (après désaimantation dans le référentiel tournant par exemple) afin que le noyau de cette intégrale ne diverge pas pour $\omega \rightarrow \omega_{0}$.

Pour effectuer ces intégrales, il suffit de balayer la fréquence de l'oscillateur suivant les lois :

$$
\begin{array}{ll}
\mathrm{d} t \propto \Delta \mathrm{d} \Delta & \text { pour } E_{\mathrm{D}} \\
\mathrm{d} t \propto \mathrm{d} \Delta / \Delta & \text { pour } \chi_{\perp} \text { où } \Delta=\omega-\omega_{0}
\end{array}
$$

qui donnent après intégration :

$$
\begin{array}{lll}
\Delta \propto \varepsilon \sqrt{|t| / \tau} & \text { pour } & E_{\mathrm{D}} \\
\Delta \propto \varepsilon \exp (|t| / \tau) & \text { pour } & \chi_{\perp}
\end{array}
$$

où $\varepsilon=+1,-1$ ou 0 selon que $t>0, t<0$ ou $t=0$.

Ces fonctions, avec les schémas des circuits générateurs, sont représentées sur la figure 9. La fonction $\exp (|t| / \tau)$ est obtenue analogiquement dans l'intervalle $(0,+\infty)$

(a)
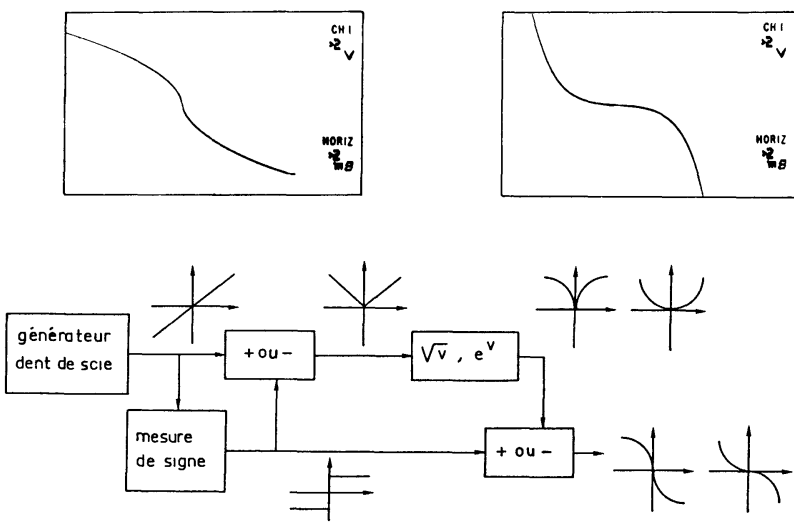

Fig. 9. - Oscillogrammes des fonctions de balayage de fréquence pour la mesure de $a$ ) $E_{\mathrm{D}}$ et $b$ ) $\chi_{\perp}$, avec le schéma simplifié de leur circuit générateur. Le bloc « + ou - » est un amplificateur inverseur ou non-inverseur selon la commande, identique à l'échantillonneur de la figure 7. Le bloc mesure de signe est également un montage standard d'amplificateur opérationnel. Les blocs $\sqrt{V}$ et exp $V$ sont des configurations appropriées d'amplificateurs logarithmiques.

Le paramètre $\tau$ est ajusté pour rendre négligeable $\int_{-\infty}^{+0} \chi^{\prime \prime}(t) \mathrm{d} t$ et par conséquent pour réduire à quelques pourcents l'erreur sur $\chi_{\perp}$. Le changement de signe pour $t=0$ est effectué par l'échantillonneur.

Pour ces intégrales et pour le calcul d'autres moments impairs, un échantillonneur à deux portes de largeur égale est préférable. L'inversion du signal à la fré- quence centrale produit le changement de signe nécessaire dans les intégrants. L'échantillonneur à trois portes décrit dans cet article servirait pour le calcul des moments pairs. Comme pour les mesures d'aires, les signaux de sortie sont insensibles aux dérives lentes de la ligne de base des $Q$-mètres. De la même façon, les intégrales sont des moyennes prises sur de nombreux cycles avec une constante de temps appropriée.

Ces instruments permettent la mesure et l'enregistrement de l'évolution dans le temps de l'énergie dipolaire, du premier moment ou déplacement de la raie, de la susceptibilité transversale et (avec des modifications évidentes) d'autres moments de la raie RMN. A titre d'exemple, nous décrivons la mesure du temps de relaxation dipolaire.

3.5 MESURE DU TEMPS DE RELAXATION DiPOLAIRE. Tout d'abord le dispositif est réglé de façon que le centre de la raie $\mathrm{RMN}$ coïncide avec le changement de signe de l'échantillonneur. Puis, l'équilibre des spins est modifié par une désaimantation dans le référentiel tournant [11], ou par une simple impulsion rf dont la fréquence est décalée par rapport au centre de la raie $\left({ }^{3}\right)$. La perturbation du système Zeeman n'est pas vue par l'appareil parce qu'elle donne une saturation symétrique de toute la raie. Par contre, la perturbation du système dipolaire produit une asymétrie dans la raie, et il apparaît un signal à la sortie du moyenneur. La décroissance de ce signal, caractérisée par le temps de relaxation dipolaire-réseau (ou dipolaire-Zeeman selon le système étudié) représente le retour de la raie à une forme symétrique. La figure 10 donne un exemple de signal dipolaire enregistré par ce procédé.

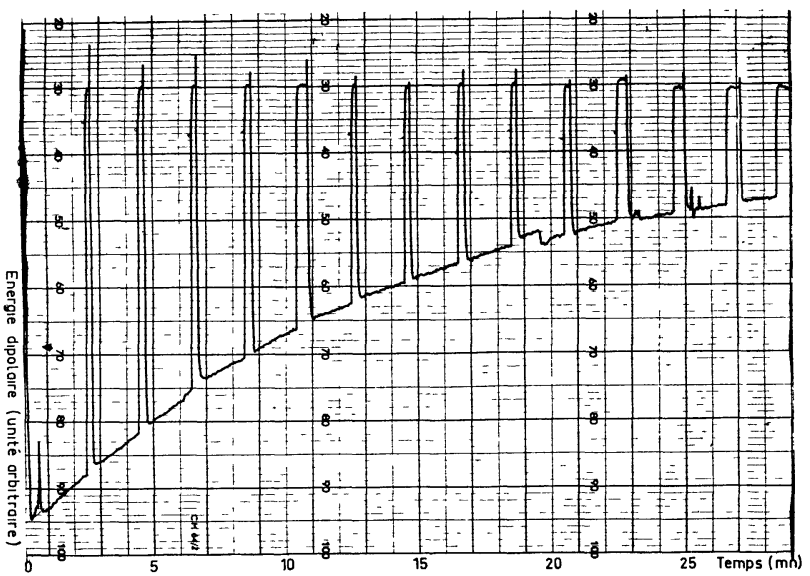

FIG. 10. - Relaxation dipolaire. La décroissance de l'intégrale $E_{\mathrm{D}}$ après une désaimantation dans le référentiel tournant est enregistrée sur la raie RMN de ${ }^{19 F}$ (dans le LiF dopé aux centres $\mathrm{F}$, à $55 \mathrm{kG}, 0,4 \mathrm{~K}$ ). Les contrôles de la ligne de base sont faits par déplacement du champ magnétique.

3.6 ERREURS SYSTÉMATIQUES ET COMPARAISON AVEC LES TECHNIQUES DIGITALES. - En conclusion, nous

(3) A comparer avec une mesure de temps dipolaire électronique [12]. 
analysons les diverses erreurs systématiques possibles dans les mesures quantitatives faites avec ces intégrateurs, en supposant d'abord que le $Q$-mètre reproduit fidèlement la composante d'absorption $\chi^{\prime \prime}(\omega)$ de la susceptibilité RMN.

La linéarité des amplificateurs de la figure $7 a$, la stabilité de leur tension de décalage et des périodes de déclenchement de la figure $7 b$ sont meilleures que $10^{-3}$ et par conséquent ne limitent pas les performances. Considérons d'abord la linéarité du balayage de fréquence, c'est-à-dire la précision avec laquelle $\omega$ suit la tension pilote $V$. Dans notre oscillateur [13] $\omega$ est déterminée par un circuit résonant dont la capacité est une diode de caractéristique $\mathrm{d}(\log C) / \mathrm{d} V=$ Cte. On peut facilement démontrer que pour un écart de fréquence $\mathrm{d} \omega$, l'erreur relative est $\mathrm{d} \omega / 2 \omega$; dans une mesure de polarisation, par exemple, l'erreur relative est de l'ordre de $\omega_{\mathrm{L}} / \omega_{0}(<0,1 \%$ pour la figure 6$)$ où $\omega_{\mathbf{L}}$ est la largeur de la raie $\mathbf{R M N}$. Dans le cas de raies (quadrupolaires) larges, cette erreur devenant importante pourrait être réduite par une compensation du circuit de polarisation de la diode (comme dans des générateurs $r$. f. commerciaux).

La troncature des ailes de la raie par le déclenchement de l'amplificateur, doit être soigneusement évitée. Pour des raies élargies simplement par interaction dipolaire entre noyaux, les ailes décroissent comme une fonction gaussienne de $\Delta=\omega-\omega_{0}$. Mais pour des échantillons dopés avec des impuretés paramagnétiques, la fréquence de résonance des noyaux proches de l'impureté se trouve déplacée plus loin dans les ailes, ce qui donne une forme lorentzienne aux ailes de la raie, variant comme $P(\Delta)=(\delta / \pi) \quad\left(\delta^{2}+\Delta^{2}\right)^{-1}\left(\right.$ où $\left.\delta=4 \pi^{2} \hbar c \gamma_{\mathrm{I}} \gamma_{\mathrm{s}} / 9 \sqrt{3}\right)$ et $c$ la concentration paramagnétique [14]. La portion de surface en dehors de $\Delta$ est donc $(8 \pi / 9 \sqrt{3}) \hbar c \gamma_{\mathrm{I}} \gamma_{\mathrm{s}} \Delta^{-1}$ et dans les conditions de la figure 6 , le calcul donne $3 \%$; cette erreur peut être réduite en augmentant l'amplitude du balayage de fréquence dans la porte centrale.

Si la fréquence de Larmor $\omega_{0}$ se trouve déplacée de $\Delta_{0}$ par rapport au centre du balayage, l'effet est nul sur la mesure de $P$. Ceci constitue un grand avantage de l'instrument. L'erreur est également nulle pour la mesure de l'énergie dipolaire $E_{\mathrm{d}}$ dans le cas d'un signal RMN dipolaire purement antisymétrique. Ceci n'est plus vrai quand le signal contient aussi une composante (Zeeman) symétrique, mais l'erreur $\left(\propto \Delta_{0} P\right)$ peut alors être identifiée comme un simple déplacement de la ligne de base puisque dans nos expériences [15], l'énergie dipolaire évolue beaucoup plus rapidement que l'énergie Zeeman. Pour la mesure de $\chi_{\perp}$, l'erreur relative est quadratique en $\Delta_{0}$, de l'ordre de $\Delta_{0}^{2} / \Delta_{\mathrm{L}}^{2}$. On peut donc toujours la rendre plus petite que l'erreur due à la troncature du signal $R M N$ à $\pm \Delta_{c}$ du centre qui est de l'ordre de $\Delta_{\mathrm{c}} / \Delta_{\mathrm{L}}$.

Pratiquement toutes ces erreurs peuvent être rendues comparables à celles provenant des termes en $\chi^{\prime \prime 2}(\omega), \chi^{\prime}(\omega)$ et $\chi^{\prime 2}(\omega)$ dans le signal du $Q$-mètre. L'effet de ces derniers sur la mesure de polarisation par exemple est décrit par Petricek et Odehnal [2] ; l'erreur dépend de la forme de la raie $R M N$ et du rapport signal sur porteuse $\mathrm{rf}$. Les termes du premier ordre en $\chi^{\prime}(\omega)$ peuvent être supprimés par un accord automatique de la fréquence ( $\$ 2$ et [5]); pour réduire l'effet des autres, nous avons travaillé sur des signaux suffisamment faibles. Ainsi quand l'amplitude du signal RMN est $5 \%$ de la porteuse r. f., l'erreur de surface provenant des termes du second ordre en $\chi^{\prime \prime}(\omega)$ n'excède pas $3 \%$ pour des formes de raie normales.

Pour des signaux sur porteuse plus grands, des corrections sont nécessaires avant l'intégration et un traitement numérique (par analyseur multicanaux ou microordinateur) est sans doute préférable. Il en est de même pour des signaux très faibles noyés dans le bruit (avec toutefois la difficulté des erreurs systématiques de quantification dans le convertisseur analogique digital). Les instruments digitaux permettent l'amélioration du rapport signal sur bruit: ils accumulent les résultats d'une série de cycles et prennent la moyenne par rapport au temps d'une quantité dans un certain intervalle. Pour un temps d'observation fixé, la technique digitale est meilleure que la technique analogique laquelle, sauf modification appropriée [16] prend une moyenne pondérée avec un poids plus grand pour les dernières informations. Mais les deux techniques fournissent des résultats équivalents pour des quantités qui varient suffisamment lentement. Par contre ce sont les instruments analogiques qui donnent la présentation la plus simple et la plus directe des quantités physiques et de l'enregistrement de leur évolution dans le temps et ceci à un prix très modique par rapport à leurs équivalents digitaux.

4. Remerciements. - Nous remercions vivement P. Deschamps, qui s'est chargé de la construction de l'échantillonneur, P. Roubeau qui a mis en œuvre un dispositif à mélange d'hélium 3 et d'hélium 4 pour la polarisation dynamique du LMN (Fig. 3 et 6), et A. Cash, H. Glattli, M. Odehnal, Y. Roinel pour des discussions utiles. La figure 8 a été enregistrée au Rutherford Laboratory.

\section{Bibliographie}

[1] Abragam, A., "The Principles of Nuclear Magnetism ", (Clarendon Press, Oxford) 1961.

[2] Petricek, V. and Odehnal, M., Nucl. Instr. Meth., 52 (1967) $197-205$.
[3] JefrRIEs, C. D., Dynamic Nuclear Orientation (Interscience Publishers) 1963.

[4] Bleaney, B. I. and Bleaney, B., Electricity and Magnetism (Clarendon Press, Oxford) 1965. 
[5] Petricek, V., Nucl. Instrum. Meth., 58 (1968) 111-116.

[6] MADDEN, DR, RPP/A 78 (1970) Rutherford Laboratory G.B.

[7] Feltin, D., 74 F 25 T Institut Laue-Langevin Grenoble (1974).

[8] Nicholas, D. J., Nanks, P. H. T., CragG, D. A., RHEL/ R 201 Rutherford Laboratory G. B. (1970)

[9] Goldman, M., Cox, S. F. J. and Bouffard, V., J. Phys. C : « Solid State Physics », 7 (1974) 2940-2952.

[10] Goldman, M., J. Mag. Res. 17 (1975) 393-8.

[11] Anderson, A. G. and Hartman, S. R., Phys. Rev., 128 (1962) 2023-2041.
[12] Cox, S. F. J., Gill, J. C. and Wharmby, D. O., J. Phys. C : « Solid State Physics » 4 (1971) 371-390.

[13] Odehnal, M., Neel, P., Bouffard, V. et Pasquette, Cl., Rev. Phys. Appl. 6 (1971) 59-63.

[14] Abragam, A., Chapellier, M., Jacquinot, F. J. and Goldman, M., J. Mag. Res. 10 (1973) 322-46.

[15] Cox, S. F. J., Bouffard, V. and Goldman, M., J. Phys. C ; Solid State Phys., 8 (1975) 3664-84.

[16] Voir par exemple PR Samways J. Phys. E : Sci Instr. 1 (1968) 142-4. 